\title{
Measuring Bias in Uncontrolled Brain Tumor Trials - to Randomize or Not to Randomize?
}

\author{
William D. Irish, David R. Macdonald, J. Gregory Cairncross
}

\begin{abstract}
Purpose: To help investigators decide if new therapies for glioma warrant definitive evaluation in randomized studies we have been developing a method for assessing the degree to which patient selection may have enhanced the results of uncontrolled treatment trials. In this study, we analyzed the impact of case selection on the survival of patients with malignant glioma receiving adjuvant stereotactic radiosurgery, a promising therapy reserved for those with small tumors and good performance status. Methods: Following published eligibility criteria we simulated the patient selection process for stereotactic radiosurgery given as a boost at the conclusion of conventional radiotherapy. Eligible patients were culled from a pre-existing clinical/imaging database of 101 consecutive conventionally-treated patients with biopsy-proven malignant glioma and known survival times. Median durations of survival and 2- and 3-year survival rates were determined for those judged eligible or ineligible for stereotactic radiosurgery. Results: Twenty-seven percent of patients were deemed eligible for stereotactic radiosurgery, eligible patients had more favorable prognostic factors and significantly longer median survival than ineligible patients ( 23.4 vs. 8.6 months; 2 -year rate, $48 \%$ vs. $15 \%$; 3 -year rate, $30 \%$ vs. $7 \%$ ); eligible patients also had a longer median survival than the entire group of unselected patients ( $23.4 \mathrm{vs.} 11.4$ months). Radiosurgery-eligible, conventionally-treated patients with glioblastoma multiforme and a group of radiosurgery-treated patients at a special referral center had similar median survival times (16.4 vs. 19.7 months). Conclusion: We provide additional evidence for selection bias in uncontrolled trials of stereotactic radiosurgery and by simulating the selection process accurately have detected a larger bias effect than noted previously. Judging from experience with interstitial radiation and intraarterial chemotherapy where substantial selection bias also occurred and randomized controlled trials proved disappointing, we conclude that a phase III study of stereotactic radiosurgery for malignant glioma is unlikely to yield a positive result and may not be necessary.
\end{abstract}

RÉSUMÉ: Mesure des biais dans les études sur les tumeurs cérébrales - randomiser ou ne pas randomiser? But: Afin d'aider les investigateurs à décider si un nouveau traitement pour le gliome mérite une évaluation définitive par des études randomisées, nous avons développé une méthode pour déterminer à quel degré la sélection des patients pourrait avoir amplifié les résultats d'essais thérapeutiques non contrôlés. Dans cette étude, nous avons analysé l'impact de la sélection des cas sur la survie des patients atteints de gliome malin, traités par radiochirurgie stéréotaxique adjuvante, une technique prometteuse réservée aux patients dont la tumeur est petite et dont l'état fonctionnel est bon. Méthodes: Conformément aux critères d'éligibilité publiés, nous avons simulé le processus de sélection des patients pour la radiochirurgie stéréotaxique administrée en bolus à la fin de la radiothérapie conventionnelle. Les patients éligibles ont été sélectionnés à partir d'une base de données cliniques et d'imagerie préexistante de 101 patients consécutifs ayant reçu le traitement conventionnel, porteurs d'un gliome malin prouvé par biopsie et dont le temps de survie était connu. La durée médiane de survie et les taux de survie à 2 et 3 ans ont été déterminés pour ceux considérés comme éligibles ou inéligibles à la radiochirurgie stéréotaxique. Résultats: Vingt-sept pourcent des patients ont été considérés comme éligibles à la radiochirurgie stéréotaxique. Les patients éligibles avaient des facteurs pronostiques plus favorables et une survie médiane significativement plus longue que les patients non éligibles. (23.4 vs. 8.6 mois; survie à 2 ans, $48 \%$ vs. $15 \%$; survie à 3 ans, $30 \%$ vs. $7 \%$ ); les patients éligibles avaient également une survie médiane plus longue que le groupe entier des patients non sélectionnés (23.4 vs. 11.4 mois). Les patients éligibles à la radiochinurgie, les patients atteints d'un glioblastome polymorphe ayant reçu le traitement conventionnel et un groupe de patients traités par radiochirurgie dans un centre de référence spécial avaient des temps de survie médians similaires (16.4 vs. 19.7 mois). Conclusions: Nos observations indiquent qu'il existe un biais de sélection dans les études non contrôlées sur la radiochirurgie stéréotaxique et, en simulant le processus de sélection avec précision, nous avons détecté un biais plus considérable que ce qui avait été noté précédemment. Selon notre expérience en ce qui concerne l'irradiation interstitielle et la chimiothérapie intra-artérielle, où des biais de sélection appréciables sont également survenus et où les essais contrôlés randomisés se sont avérés décevants, nous concluons qu'une étude de phase III sur la radiochirurgie stéréotaxique dans le gliome malin est peu susceptible de donner un résultat positif et n'est peut-être pas nécessaire.

Can. J. Neurol. Sci. 1997; 24: 307-312

Patients with malignant glioma destined to have better than expected outcomes often are selected to receive new therapies. Interpretative difficulties arise: are good results due to careful patient selection, novel treatment, or some combination of the two $?^{1-3}$ To help investigators decide if new therapies for glioma warrant definitive evaluation in randomized studies we have been developing a method for assessing the degree to which patient selection may have enhanced the results of uncontrolled treatment trials. ${ }^{2,3}$ Our method relies on 1) a clinical/imaging database of unselected, consecutive, conventionally-treated patients with known outcomes and 2) a sufficiently detailed

From the Pittsburgh Transplantation Institute, University of Pittsburgh, Pittsburgh, Pennsylvannia (W.D.I); Departments of Clinical Neurological Sciences and Oncology, University of Western Ontario, (D.R.M., J.G.C.), and London Regional Cancer Centre, London, Ontario (D.R.M., J.G.C.).

RECEIVED DECEMBER 16, 1996. ACCEPTED IN FINAL FORM APRIL 4, 1997.

Reprint requests to: J. Gregory Cairncross, London Regional Cancer Centre. 790 Commissioners Road, East, London, Ontario, Canada N6A 4L6 
description of the eligibility criteria for the novel therapy that the selection process can be reenacted closely and a matched group of patients culled from the database. Predicted outcomes for the simulated "control" group then can be compared to reported outcomes for the "experimental" group and the relative contributions of patient selection and treatment prescription evaluated; similar outcomes point to substantial selection bias while differences favoring the experimental group suggest a therapeutic effect. Matched historical controls are a suitable comparative group for an illness like glioma because survival times worldwide have not changed in several decades. Using this approach, we evaluated a new therapy for malignant glioma called stereotactic radiosurgery.

Stereotactic radiosurgery is considered a promising adjunctive therapy for patients with malignant glioma $;{ }^{4}$ small residual tumor volumes visible by imaging at the conclusion of standard external beam radiotherapy receive supplemental radiation by this precision focused single-dose method. Loeffler et al. treated 69 patients with glioblastoma multiforme with conventional radiation followed by a radiosurgery boost and reported a median survival time of 19.7 months. ${ }^{5}$ Since patients with glioblastoma multiforme live on average six to 10 months following standard treatment these results may represent a substantial two- to three-fold increase in longevity. The following analysis of consecutive, conventionally-treated patients with malignant glioma was designed to address the following questions: what proportion of patients would have been eligible for stereotactic radiosurgery; did eligible patients live longer than ineligible patients; did eligible patients live longer than expected; did eligible patients live as long as a comparable group of patients receiving radiosurgery; and among eligible patients, what were the relative contributions to survival of small tumor size and good performance status?

\section{Methods}

This analysis relied on a database assembled by Florell and colleagues. ${ }^{2}$ Briefly, Florell et al. reviewed the records of all newly diagnosed patients with biopsy-proven supratentorial malignant glioma treated at the London Regional Cancer Centre in London, Ontario, from 01 January 1988 to 31 December 1989 , choosing a center where experience with malignant glioma is population-based to minimize bias introduced by preselection of cases by referring doctors. [Population of referral-base $1.6 \mathrm{mil}$ lion; incidence of malignant glioma 6 per 100,000 population per year; expected number of cases over two years - 96; observed number of cases 101.] They also chose a center where patients received standard initial treatment consisting of maximal feasible surgical resection followed by external beam radiotherapy. Most patients also received adjuvant systemic chemotherapy, either carmustine (BCNU) or combination chemotherapy containing a nitrosourea. Treatment for recurrent tumor was individualized although none had radiosurgery. Adrenocorticosteroids were used perioperatively, during the early stages of radiotherapy, and as necessary thereafter, but not used to prolong terminal situations. Financial considerations were not a barrier to diagnosis, treatment, or follow-up. Data abstracted from each patient's clinical record included date of diagnosis, age at diagnosis, tumor type (glioblastoma multiforme or anaplastic glioma), Karnofsky Performance Status (KPS) before surgery, after surgery and at the end of radiotherapy, and date and cause of death. Postoperative computerized tomography (CT) scans obtained within 96 hours of surgery were compared to preoperative scans and the degree of resection of the enhancing mass estimated as follows: biopsy only; minor resection, $<25 \%$ removed; partial resection, $25-49 \%$ removed; major resection, $50-90 \%$ removed; near total resection, $>90 \%$ removed. For non-enhancing tumors, the degree of resection of the abnormal area was estimated. Postradiotherapy CT scans were reviewed and the largest diameter of any residual tumor measured.

Retrospectively and blind to outcome, we judged each patient in the database eligible or ineligible for stereotactic radiosurgery following guidelines set out for this new therapy by Loeffler et al. ${ }^{4}$ Specifically, patients with large tumors (maximum diameter $>4 \mathrm{~cm}$ ) on the postradiation scan, or no visible tumor after radiation, or no identifiable target (e.g., lobectomy with gross total resection) were deemed ineligible as were those with a postradiation KPS $<70$. Patients with tumors originating in the brain stem, near the optic chiasm (within $5 \mathrm{~mm}$ ) or spreading subependymally also were excluded. Age and other health problems were not considered in making eligible/ineligible assignments. Survival was measured from the date of diagnosis. For the entire group, the subset with glioblastoma multiforme and the subset with anaplastic glioma, survival curves for eligible and ineligible patients were generated using the product-limit method (Kaplan-Meier) ${ }^{6}$ and median survivals compared using the generalized Wilcoxon (Breslow) test. ${ }^{7}$ Patients alive on 10 October 1995 were censored, but four dying early on due to pulmonary embolism were included. Eligible and ineligible patients were compared with respect to age extent of resection, and KPS before and after surgery and after radiotherapy: mean age and mean KPS were compared using the student's T-test; analysis of covariance was used to compare changes in KPS that occurred after surgery and radiotherapy; and degrees of resection were compared using the chi-square test. All $P$ values were based on two-tailed tests. The relative contributions to survival of postradiation tumor volume and postradiation performance status were assessed as follows: survival curves were generated and median survivals calculated after waiving the restriction on tumor size, while maintaining KPS criteria, and then again after waiving the restriction on KPS, maintaining tumor size criteria.

\section{RESULTS}

One hundred and one patients ( 54 men, 47 women) with supratentorial malignant glioma were seen at a regional cancer center in Canada over two years (1988 and 1989). They ranged in age from 16-81 years (mean, 54 years). The diagnosis was glioblastoma multiforme in 68 patients and anaplastic glioma in 33 (27 anaplastic astrocytomas, five anaplastic mixed gliomas, and one anaplastic oligodendroglioma). Thirty-three patients had frontal tumors, 31 temporal, 18 parietal, three occipital, two thalamic, 11 bihemispheric or callosal, and three multifocal. The tumor was right-sided in 53 patients and left-sided in 35. Sixteen patients had a biopsy only, 19 a minor resection, 15 a partial resection, 30 a major resection, 20 a near total resection, and one patient was not evaluable in this respect because the postoperative CT scan was missing, but based on the operative note had a near total resection. Eighty patients completed radiotherapy, two refused radiation, 10 were not radiated due to poor postoperative functional status, and radiation was discontinued in nine patients whose condition deteriorated. The mean KPS was 70.3 before surgery, 70.1 after surgery, and 70.7 for those 
completing radiotherapy. At the completion of radiotherapy, 54 patients had a KPS $\geq 70,33$ had visible residual tumor with a maximum diameter $\leq 4 \mathrm{~cm}$ (mean, $3.1 \mathrm{~cm}$ ), and 27 had both characteristics, that is high performance status and small tumor burden. Median survival was 11.4 months for all patients, 8.8 months for those with glioblastoma multiforme, and 27.0 months for those with anaplastic glioma.

Overall, 27 patients (27\%) were deemed eligible for stereotactic radiosurgery and 74 were judged ineligible; the clinical features of both groups are summarized in Table 1 . The median duration of survival for eligible patients was 23.4 months $(95 \%$ confidence interval, 19.0 to 28.0 months) compared to 8.6 months (95\% confidence interval, 5.4 to 11.8 months) for ineligible patients $(P<0.00001$, Figure 1). Eligible patients were younger $(P<0.0001)$, had more extensive resections $(P=$ $0.003)$ and had better function before and after surgery and after radiation $(P$ values $<0.0001)$ than their ineligible counterparts. Significant differences in postoperative and postradiation KPS scores between eligible and ineligible patients remained after adjusting for preoperative differences in performance status
(Table 1). Eighteen patients with glioblastoma multiforme $(26 \%)$ were deemed eligible for stereotactic radiosurgery and 50 were judged ineligible (Table 2). The median duration of survival for eligible patients with glioblastoma multiforme was 16.4 months (95\% confidence interval, 8.9 to 23.9 months) compared to 6.6 months ( $95 \%$ confidence interval, 4.5 to 8.8 months) for ineligible patients $(P=0.0001$, Figure 2$)$. Eligible patients were younger $(P=0.001)$, had more extensive resections $(P=0.02)$ and had better function before and after surgery and after radiation $(P$ values $<0.0001)$ than their ineligible counterparts. Significant differences in postradiation KPS scores between eligible and ineligible patients remained after adjusting for preoperative differences in performance status (Table 2). Nine patients with anaplastic glioma $(27 \%)$ were deemed eligible for stereotactic radiosurgery and 24 were judged ineligible. The median duration of survival for eligible patients with anaplastic glioma has not been reached but was 14.8 months (95\% confidence interval, 0 to 32.3 months) for ineligible patients $(P=0.003)$. The median durations of survival and the two- and three-year survival rates are summarized in Table 3.

Table 1: Characteristics of 101 Eligible and Ineligible Patients ${ }^{\mathrm{a}}$.

\begin{tabular}{|c|c|c|c|c|}
\hline & $\begin{array}{l}\text { CLINICAL } \\
\text { VARIABLE }\end{array}$ & $\begin{array}{l}\text { ELIGIBLE } \\
(\mathbf{n}=27)\end{array}$ & $\begin{array}{l}\text { INELIGIBLE } \\
(n=74)\end{array}$ & $\begin{array}{l}\text { SIGNIFICANCE } \\
\text { (p value) }\end{array}$ \\
\hline Age (years) & & $46.1 \pm 13.2$ & $57.8 \pm 14.4$ & $<0.0001$ \\
\hline Sex (M/F ratio) & & $16 / 11$ & $38 / 36$ & 0.481 \\
\hline \multirow[t]{5}{*}{ Surgery } & biopsy & $1(4 \%)$ & $15(20 \%)$ & $0.003^{\mathrm{b}}$ \\
\hline & $\min 0 r(<25 \%)$ & $2(7 \%)$ & $17(23 \%)$ & \\
\hline & partial $(25-49 \%)$ & $4(15 \%)$ & $11(15 \%)$ & \\
\hline & major $(50-90 \%)$ & $8(30 \%)$ & $22(30 \%)$ & \\
\hline & near total $(>90 \%)$ & $12(44 \%)$ & $9(12 \%)$ & \\
\hline \multirow[t]{2}{*}{ Pathology } & glioblastoma & $18(67 \%)$ & $50(68 \%)$ & 0.932 \\
\hline & anaplastic glioma & $9(33 \%)$ & $24(32 \%)$ & \\
\hline \multirow[t]{3}{*}{ KPS } & before surgery & $80.7 \pm 13.0$ & $66.2 \pm 17.3$ & $<0.0001$ \\
\hline & after surgery & $81.9 \pm 13.0$ & $64.6 \pm 18.0$ & $<0.0001(0.020)^{\mathrm{c}}$ \\
\hline & postradiation & $80.7 \pm 9.6$ & $65.3 \pm 14.8^{\mathrm{d}}$ & $<0.0001(0.001)^{\mathrm{c}}$ \\
\hline Diameter $(\mathrm{cm})$ & postradiation & $3.1 \pm 0.8$ & $5.2 \pm 1.0^{\mathrm{e}}$ & $<0.0001$ \\
\hline
\end{tabular}

"Mean values \pm standard error ${ }^{b}$ Exact significance ${ }^{c}$ Analysis of covariance $f$-test

${ }^{\mathrm{d}}$ Mean score in 53, excludes 21 patients not having or completing radiation

${ }^{\mathrm{e}}$ Mean value in 46 , excludes 21 not having or completing radiation and 7 with multifocal/diffuse lesions.

Table 2: Characteristics of Patients With Glioblastoma Multiforme".

\begin{tabular}{lllll}
\hline & $\begin{array}{l}\text { CLINICAL } \\
\text { VARIABLE }\end{array}$ & $\begin{array}{l}\text { ELIGIBLE } \\
(\mathbf{n}=\mathbf{1 8})\end{array}$ & $\begin{array}{l}\text { INELIGIBLE } \\
(\mathbf{n}=\mathbf{5 0})\end{array}$ & $\begin{array}{l}\text { SIGNIFICANCE } \\
\text { (p value) }\end{array}$ \\
\hline Age (years) & & $50.2 \pm 13.8$ & $61.7 \pm 10.6$ & $0.001^{\mathrm{b}}$ \\
Sex (M/F ratio) & & $10 / 8$ & $26 / 24$ & 0.796 \\
Surgery & biopsy & $1(6 \%)$ & $11(22 \%)$ & $0.022^{\mathrm{b}}$ \\
& minor (<25\%) & $2(11 \%)$ & $10(20 \%)$ & \\
& partial (25-49\%) & $6(33 \%)$ & $7(14 \%)$ & $<0.0001$ \\
& major (50-90\%) & $9(50 \%)$ & $9(18 \%)$ & $<0.0001(0.107)^{\mathrm{c}}$ \\
KPS & near total (>90\%) & $80.0 \pm 12.4$ & $63.6 \pm 16.9$ & $<0.0001(0.028)^{\mathrm{c}}$ \\
\hline
\end{tabular}

aMean values \pm standard error bxact significance ${ }^{\mathrm{b}}$ Analysis of covariance $\mathrm{f}$-test

dMean score in 33, excludes 17 patients not having or completing radiation. 
Table 3: Durations of Survival for Eligible/Ineligible Patients.

\begin{tabular}{|c|c|c|c|c|c|c|}
\hline \multirow[t]{2}{*}{$\begin{array}{l}\text { SURVIVAL } \\
\text { DATA }\end{array}$} & \multicolumn{2}{|c|}{ ALL PATIENTS } & \multicolumn{2}{|c|}{$\begin{array}{c}\text { GLIOBLASTOMA } \\
\text { MULTIFORME }\end{array}$} & \multicolumn{2}{|c|}{$\begin{array}{c}\text { ANAPLASTIC } \\
\text { GLIOMA }\end{array}$} \\
\hline & Eligible & Ineligible & Eligible & Ineligible & Eligible & Ineligible \\
\hline Median (mos) & 23.4 & 8.6 & 16.4 & 6.6 & N/A & 14.8 \\
\hline 2-Year (\%) & 48 & 15 & 28 & 0 & 89 & 46 \\
\hline 3-Year $(\%)$ & 30 & 7 & 6 & 0 & 78 & 21 \\
\hline
\end{tabular}

$\mathrm{N} / \mathrm{A}=$ not applicable

Thirty-three patients, 23 with glioblastoma multiforme and ten with anaplastic glioma, would have been eligible for stereotactic radiosurgery based solely on tumor size, but six with small tumors (five with glioblastoma multiforme) were rendered ineligible by a postradiation KPS $<70$. Patients with small tumors after conventional radiation had a median duration of survival of 19.8 months (95\% confidence interval, 12.5 to 27.1 months) compared to 8.6 months (95\% confidence interval, 5.3 to 12.0 months $)$ for those with large tumors $(P=0.0002)$ and compared to 23.4 months for radiosurgery-eligible patients. Patients with glioblastoma multiforme and small tumors had a median duration of survival of 15.8 months (95\% confidence interval, 10.8 to 20.8 months) compared to 6.6 months (95\% confidence interval, 4.8 to 8.4 months) for those with large tumors $(P=0.0003)$ and compared to 16.4 months for eligible patients (Figure 2). Fifty-four patients, 33 with glioblastoma multiforme and 21 with anaplastic glioma, would have been eligible for stereotactic radiosurgery based solely on postradiation performance status criteria, but 27 with a postradiation KPS $\geq$ 70 (15 with glioblastoma multiforme) were rendered ineligible by large tumor size. Patients with good performance status had a median duration of survival of 21.8 months $(95 \%$ confidence

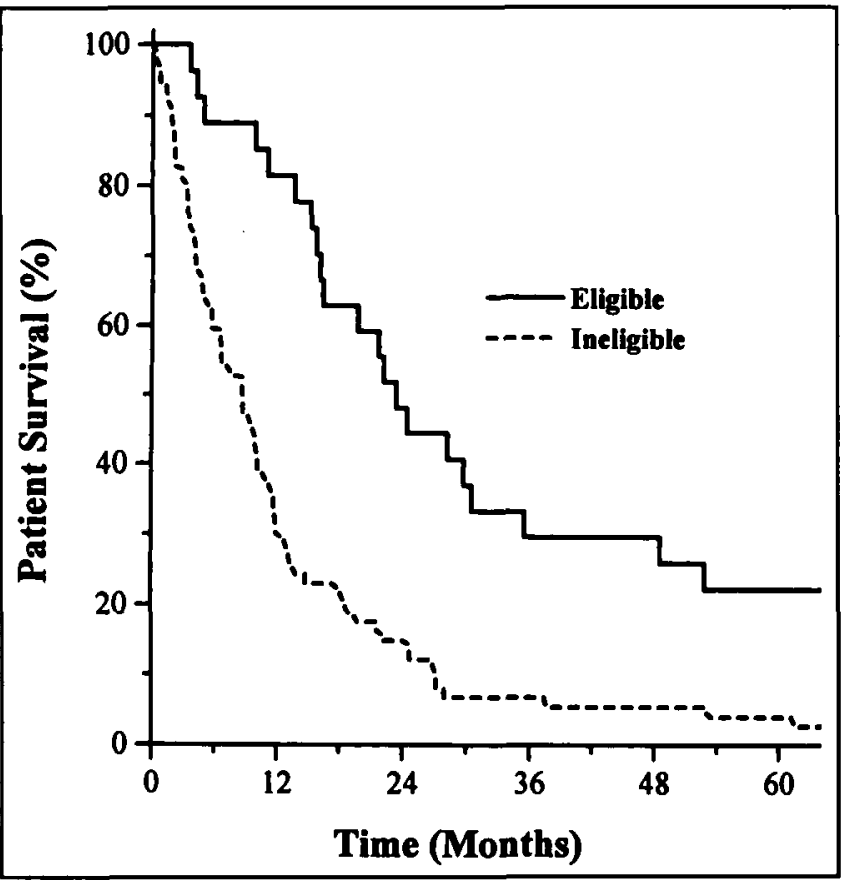

Figure 1: Survival curves for all patients deemed eligible $(n=27)$ or ineligible $(n=74)$ for stereotactic radiosurgery. interval, 17.0 to 26.6 months) compared to 4.7 months (95\% confidence interval, 3.3 to 6.0 months) for those with poor performance scores $(P<0.00001)$ and compared to 23.4 months for radiosurgery-eligible patients. Patients with glioblastoma multiforme and KPS $\geq 70$ had a median duration of survival of 13.8 months (95\% confidence interval, 9.0 to 18.6 months) compared to 4.1 months (95\% confidence interval, 1.8 to 6.3 months) for those with KPS $<70(P<0.00001)$ and compared to 16.4 months for eligible patients.

Lastly, we repeated the analysis excluding 12 patients (all ineligible) who refused radiation (two patients) or were not irradiated due to poor postoperative performance status ( 10 patients). The median duration of survival for all ineligible patients was then 9.9 months (95\% confidence interval, 8.2 to 11.6 months) and for the subset of ineligible patients with glioblastoma multiforme was 6.7 months (95\% confidence interval, 4.4 to 9.0 months). Both durations of survival were significantly shorter than those observed for eligible patients ( $p$ values, 0.0001 and 0.0002 respectively).

\section{Discussion}

Curran and colleagues ${ }^{8}$ in a reanalysis of survival data pooled from three randomized controlled trials conducted over two decades by the Radiation Therapy Oncology Group (RTOG) concluded that pretreatment characteristics, moreso than current treatment, were the major predictors of outcome for patients with malignant glioma and that survival results could be profoundly influenced by the prognostic factor profile of the trial's participants. Curran et al. ${ }^{8}$ and an earlier report by Florell et al. ${ }^{2}$ focused attention on the relationship between patient selection for a novel therapy and survival results after treatment, paving the way for this analysis of stereotactic radiosurgery. Loeffler

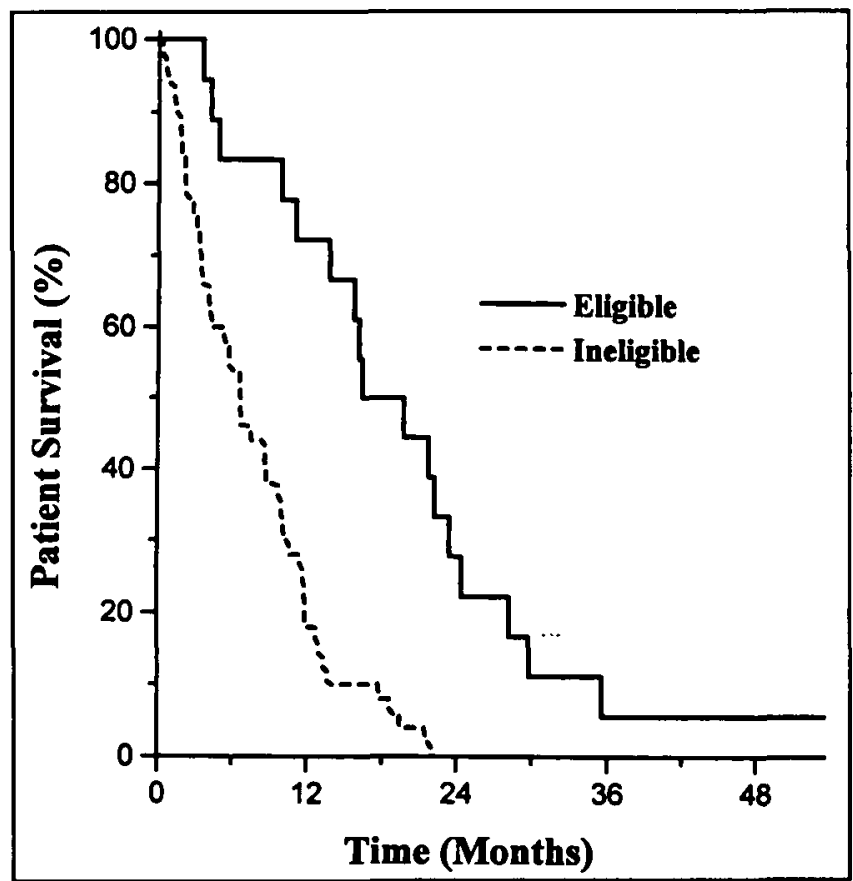

Figure 2: Survival curves for patients with glioblastoma multiforme deemed eligible $(n=18)$ or ineligible $(n=50)$ for stereotactic radiosurgery. 
et al. ${ }^{4}$ described in detail how patients at the Joint Center for Radiation Therapy in Boston were selected for a stereotactic radiosurgery boost administered at the conclusion of conventional external beam radiotherapy; given these well defined eligibility criteria ${ }^{4}$ and a clinical database of consecutive conventionally-treated patients with known outcomes that also included postradiation imaging studies, ${ }^{2}$ we were able to simulate closely the patient selection process for this new glioma therapy. Postradiation images were critical to this exercise because this is the timepoint at which patients with glioblastoma multiforme treated by Loeffler et al. ${ }^{4}$ were judged to be eligible or ineligible for radiosurgery. In this analysis, $26 \%$ of patients with glioblastoma multiforme were deemed eligible for stereotactic radiosurgery, eligible patients lived significantly longer than ineligible patients by virtue of younger age, larger tumor resections, smaller tumor burdens and better performance status (16.4 vs. 6.6 months), eligible patients lived twice as long as the entire group of unselected patients (16.4 vs. 8.8 months) and eligible patients had a median duration of survival that was not significantly different from those actually receiving a stereotactic radiosurgery boost (16.4 vs. 19.7 months). ${ }^{5}$ Both small tumor size (postradiation) and good performance status (postradiation) were important prognostic factors and together contributed to the superior survival of radiosurgery-eligible cases.

Curran and colleagues ${ }^{9}$ in a study similar to this one reported that $12 \%$ of newly diagnosed patients were eligible for radiosurgery and that eligible patients lived longer than ineligible patients (14.4 vs. 11.7 months; 12.5 vs. 10.5 months for those with glioblastoma multiforme). The authors concluded that prognostic factors, especially performance status, contributed to the longer survival of radiosurgery-eligible patients and called for definitive evaluation of stereotactic radiosurgery in a randomized controlled trial. Although the differences in median survival between eligible and ineligible patients in the analysis by Curran et al..$^{9}$ were statistically significant, radiosurgery-eligible patients in that study had a shorter median duration of survival than did radiosurgery-eligible patients in our study (14.4 vs. 23.4 months; 12.5 vs. 16.4 months for those with glioblastoma multiforme). As distinct from Loeffler et al. ${ }^{4}$ and ourselves, Curran et al. ${ }^{9}$ based eligibility for stereotactic radiosurgery on preoperative CT scans and postoperative KPS rather than postradiation images and postradiation performance status. By approximating the selection process rather than imitating it, Curran et al. ${ }^{9}$ may have underestimated the median duration of survival of eligible patients and also underestimated the survival advantage of eligible patients compared to ineligible patients. Furthermore, Curran et al. ${ }^{9}$ chose eligible patients from a group of preselected patients (i.e., RTOG study participants) whereas we chose them from a group of consecutive unselected patients at a center where experience with glioma is populationbased. Methodological differences such as these may not be inconsequential: Kirby et $a .^{3}$ demonstrated that the survival of patients judged eligible for intraarterial chemotherapy varied with the selection criteria used; by some criteria, eligible patients lived significantly longer than ineligible patients, but by other criteria, eligible and ineligible cases had similar survival times. ${ }^{3}$ Paradoxically, our group of radiosurgery-eligible patients, a much larger proportion of all patients, lived longer not shorter than the radiosurgery-eligible group reported by Curran et al. ${ }^{9}$
Sarkaria and colleagues ${ }^{10}$ in another study analyzing the impact of patient selection on the results of stereotactic radiosurgery concluded that patients receiving a radiosurgery boost lived significantly longer than "matched" historical controls from the RTOG database. ${ }^{8}$ However, imaging studies were not reviewed, and for this reason Sarkaria et al. ${ }^{10}$ had no way of knowing whether control patients culled from the RTOG database had small tumors and would have been eligible for stereotactic radiosurgery based on tumor size criteria; indeed, one can infer from Curran et al. ${ }^{9}$ and our analysis that most did not and were not. Again, by approximating the selection process for stereotactic radiosurgery rather than modeling it, Sarkaria et al. ${ }^{10}$ selected a suboptimal control group and as a consequence may have underestimated the median duration of survival of eligible patients. Sarkaria et al. ${ }^{10}$ called for definitive evaluation of stereotactic radiosurgery for malignant glioma in a randomized controlled trial.

It is clear from these studies ${ }^{9,10}$ that patient selection contributes to long survival after stereotactic radiosurgery, but what is the magnitude of this effect? In this study, we provide additional evidence for selection bias in uncontrolled trials of stereotactic radiosurgery but by simulating the selection process more closely appear to have detected a larger bias effect than noted previously. Patients in the radiosurgery-treated experimental group reported by Loeffler et al. ${ }^{5}$ lived slightly longer than patients in the radiosurgery-eligible control group culled from our database (19.7 vs 16.4 months) but other sources of selection bias not accounted for in our analysis might explain this modest survival difference. Although we attempted to make the experimental and control groups similar by reenacting the selection process exactly, patients treated with stereotactic radiosurgery may have had more intensive treatment at recurrence or other important characteristics, not easily modeled, which contributed to their longer survival. For example, the experimental group of Loeffler et al. ${ }^{5}$ may have had a higher rate of reoperation, fewer comorbid conditions, or less aggressive tumors than the simulated control group. The rate of reoperation was $30 \%$ in our radiosurgery-eligible patients but will be as high as $50 \%$ following stereotactic radiosurgery (JS Loeffler, personal communication). It is common practice in neuro-oncology to exclude from trials of new therapies patients with other serious medical conditions that could jeopardize survival, and it is conceivable that the referral process to special brain tumor centers selects for novel treatment a group of patients with less aggressive gliomas destined to live longer. Furthermore, we observed previously that patients at our center who participated in a randomized controlled trial lived $20 \%$ longer than patients who were study-eligible but refused to participate'; volunteers often are highly motivated and in some circumstances might be expected to live longer. The median survival of the simulated control group in our analysis would be underestimated if these additional sources of bias were present and unaccounted for in a modeling paradigm.

Although radiosurgery-eligible patients in this study were selected from only 101 total cases, the database compiled by Florell et al. ${ }^{2}$ is well suited to this type of analysis. The database reflects a population-based experience (see Methods) and 
includes contemporary consecutive patients all of whom received uniform standard initial therapy for pathologicallyverified malignant glioma. Subsequently, all patients were followed closely by two experienced clinicians and reimaged at predetermined timepoints, including postradiation.

Our data suggest that bias, moreso than treatment, explains the long durations of survival after stereotactic radiosurgery and leads us to question whether a randomized controlled trial is truly necessary. As we interpret the data, a phase III study of stereotactic radiosurgery as currently prescribed for malignant glioma is unlikely to yield a positive result. The validity of this type of analysis as a predictor of the results of randomized comparisons has yet to be established, but when intraarterial chemotherapy was modeled, eligible patients lived much longer than expected ${ }^{3}$ and the randomized controlled trial was negative ${ }^{11}$ and similarly, when interstitial radiation was modeled, eligible patients lived much longer than expected ${ }^{2}$ and the randomized controlled trial demonstrated a great deal less benefit than anticipated..$^{12}$ Expense and neurotoxicity were important features of the experimental arms of the randomized trials evaluating both interstitial radiation and intraarterial chemotherapy and will be encountered again with radiosurgery. High rates of steroid dependence, high rates of reoperation for radionecrosis and high cost will detract from any modest survival benefit that may ensue from stereotactic radiosurgery.

While there may be no substitute for randomized controlled trials, exercises like this one, by disclosing bias, may help investigators interpret uncontrolled trials of new therapies for glioma, priorize novel treatments for phase III evaluation, or better estimate outcomes and variance for power calculations for subsequent randomized trials. We analyzed survival following selection for radiosurgery but this modeling approach could be adapted to assess other outcomes, including for example quality of life, provided the reference database also included quality of life information. Clinicians may find simulated control techniques particularly valuable when there are many new therapies to be tested but limited resources for patient-based research, especially clinical trials.

\section{ACKNOWLEDGEMENTS}

The authors thank Dr. Randall Florell for compiling the database, Dr. Jerome Posner for reviewing the manuscript and Pat Gray for preparing it.

\section{REFERENCES}

1. Winger MJ, Macdonald DR, Schold SC, Cairncross JG. Selection bias in clinical trials of anaplastic glioma. Ann Neurol 1989; 26: 531-534.

2. Florell RC, Macdonald DR, Irish WD, et al. Selection bias, survival, and brachytherapy for glioma. J Neurosurg 1992; 76: 179-183.

3. Kirby S, Brothers M, Irish W, et al. Evaluating glioma therapies modeling treatments and predicting outcomes. J Natl Cancer Inst 1995; 87: 1884-1888.

4. Loeffler JS, Alexander E, Shea WM, et al. Radiosurgery as part of the initial management of patients with malignant gliomas. J Clin Oncol 1992; 10: 1379-1385.

5. Loeffler JS, Shrieve DC, Wen PY, et al. Radiosurgery for intracranial malignancies. Sem Radiat Oncol 1995; 5: 225-234.

6. Kaplan EL, Meier P. Non-parametric estimation from incomplete observations. J Am Stat Assoc 1958; 53: 437-481.

7. Breslow NE. A generalized Kurskel-Wallis test for comparing $\mathrm{K}$ samples subject to unequal patterns of censorship. Biometrika 1970; 57: 579-594.

8. Curran WJ, Scott CB, Horton J, et al. Recursive partitioning analysis of prognostic factors in three Radiation Therapy Oncology Group malignant glioma trials. J Natl Cancer Inst 1993; 85: 704-710.

9. Curran WJ, Scott CB, Weinstein AS, et al. Survival comparison of radiosurgery-eligible and -ineligible malignant glioma patients treated with hyperfractionated radiation therapy and carmustine: a report of Radiation Therapy Oncology Group 83-02. J Clin Oncol 1993; 11: 857-862.

10. Sarkaria MD, Mehta MP, Loeffler JS, et al. Radiosurgery in the initial management of malignant gliomas: survival comparison with the RTOG recursive partitioning analysis. Int J Radiat Oncol Biol Phys 1995; 32: 931-941.

11. Shapiro WR, Green SB, Burger PC, et al. A randomized comparison of intra-arterial versus intravenous $\mathrm{BCNU}$, with or without intravenous 5-fluorouracil, for newly diagnosed patients with malignant glioma. J Neurosurg 1992; 76: 772-781.

12. Shapiro WR, Green $S$, Burger $P$, et al. A randomized trial of interstitial radiotherapy (IRT) boost for the treatment of newly diagnosed malignant glioma (glioblastoma multiforme, anaplastic astrocytoma, anaplastic oligodendroglioma, malignant mixed glioma): BTCG Study 8701. Neurology 1994; 44 (Suppl 2): A263. 